\title{
Comparison of impact of target delineation of computed tomography- and magnetic resonance imaging-guided brachytherapy on dose distribution in cervical cancer
}

\author{
Lalida Tuntipumiamorn, MSc', Suphalerk Lohasammakul, MD², Pittaya Dankulchai, MD', Pitchayut Nakkrasae, MSc' \\ 'Division of Radiation Oncology, Department of Radiology, ${ }^{2}$ Department of Surgery, Faculty of Medicine, Siriraj Hospital, Mahidol University, \\ Bangkok, Thailand
}

\begin{abstract}
Purpose: The dose distributions obtained from three imaging approaches for target delineation in cervical cancer using high-dose-rate (HDR) brachytherapy were investigated.

Material and methods: Ten cervical cancer patients receiving four fractions of HDR brachytherapy were enrolled. Based on different imaging approaches, three brachytherapy plans were developed for each patient: with the high-risk clinical target volume (HRCTV) delineated on magnetic resonance (MRI) images for every fraction (approach A; MRIonly); on MRI for the first fraction and computed tomography (CT) images for the subsequent fractions (approach B; $\mathrm{MRI}_{1 \mathrm{st}} / \mathrm{CT}$ ); and on CT images for all fractions (approach C; CT-only). The volume, height, width at point A, width at maximum level, and dosimetric parameters $\left(\mathrm{D}_{100}, \mathrm{D}_{98}, \mathrm{D}_{95}\right.$, and $\mathrm{D}_{90}$ of the HRCTV; and $\mathrm{D}_{0.1 \mathrm{cc}}, \mathrm{D}_{1 \mathrm{cc}}$, and $\mathrm{D}_{2 \mathrm{cc}}$ of all organs at risk, or organ at risk - OAR: bladder, rectum, sigmoid colon, and bowel) provided by each approach were compared.

Results: The mean HRCTV volume, width, and height obtained from approach C (CT-only) were overestimated compared to those from approaches $\mathrm{A}$ (MRI-only) and B $\left(\mathrm{MRI}_{1 \mathrm{st}} / \mathrm{CT}\right)$. The doses to the HRCTV for approaches A and B were similar. However, the HRCTV doses for approach $C$ were significantly lower than those for approaches A and B for all parameters $\left(D_{95}-D_{100}\right)$. As to the OAR, the three approaches showed no differences.

Conclusions: A combination of MRI and CT is a safe alternative approach for cervical cancer HDR brachytherapy. The technique provides comparable dosimetric outcomes to MRI-based planning, while being more cost-effective.

Key words: brachytherapy, cervix cancer, CT, MRI, target delineation.

\section{Purpose}

High-dose-rate (HDR) brachytherapy (BT) plays an essential role in the treatment of invasive cervical cancer $[1,2]$. With current technologies, the planning images have advanced from 2-dimensional-based planning to 3-dimensional image-guided brachytherapy (3D IGBT) $[3,4,5,6]$. The treatment delivery can be guided by either computed tomography (CT) or magnetic resonance imaging (MRI), but MRI has been demonstrated to provide superior soft-tissue contrast compared to $\mathrm{CT}$, better image of extension of primary tumor, and its relationship with organs at risk is evidenced $[7,8,9,10,11,12]$. GEC-ESTRO after employment of MRI in cervix brachytherapy, with institutional series of hundreds of patients, EMBRACE study, and RetroEMBRACE study, have demonstrated better local controls and survival in these patients, when
BT treatment is defined in a volume not in point $\mathrm{A}$, and with the use of MRI $[13,14,15]$. However, the availability of MRI-based BT is limited at some radiotherapy centers from time, labor, and resource intensive. Transition to MRI-based BT as more accessible for institutions, with safe and efficient accomplishment was described by Harkenrider et al. [16].

Given these limitations, the application of planning involving a combination of MRI and CT has been proposed $\left(\mathrm{MRI}_{1 \mathrm{st}} / \mathrm{CT}\right)$. With this approach, the MRI is performed only in the first fraction of the treatment for target and organ at risk (OAR) delineation, applicator reconstruction, and dose optimization $[17,18]$. The contour information is then used to guide the radiation oncologist in determining the tumor target in the subsequent fraction by using CT images. The present research was therefore conducted to compare the impacts of the target

\footnotetext{
Address for correspondence: Lalida Tuntipumiamorn, MSc, Division of Radiation Oncology, Department Received: 19.07 .2018 of Radiology, Faculty of Medicine, Siriraj Hospital, Mahidol University, 2 Prannok Rd, 10700 Bangkoknoi, Accepted: 25.09.2018 Thailand, phone: +66 944970909, 凶 e-mail: lalida.tun@mahidol.ac.th or 1.tunti@gmail.com

Published: 30.10 .2018
} 
delineation accuracies obtained using imaging with CT only, MRI only, and $\mathrm{MRI}_{1 \mathrm{st}} / \mathrm{CT}$ on the dose distributions in cervical cancer brachytherapy planning.

\section{Material and methods}

\section{Patient selection}

The study was approved by the Siriraj Institutional Review Board, Faculty of Medicine, Siriraj Hospital, Mahidol University (project number 760/2560). Between August 2015 and December 2016, 10 cervical cancer patients with stage IB2 to IIIB tumors were enrolled. All patients received 50 Gy external beam radiotherapy to the whole pelvis prior to the brachytherapy. The HDR brachytherapy schedule consisted of 4 treatment fractions with prescribed dose of 7 Gy. Intracavitary radiation therapy, using MRI-compatible tandem/ring applicators with or without interstitial plastic needles (Varian Medical Systems, Palo Alto, CA, USA) was performed for each treatment application.

\section{Image acquisition}

Patients with an applicator underwent T2-weighted MR imaging, with a $3 \mathrm{~mm}$ slice thickness using a 1.5-Tesla MRI scanner (Philips Archiva, Philips Medical Systems B.V., Eindhoven, The Netherlands) and CT imaging with a $1.25 \mathrm{~mm}$ slice thickness (GE Brightspeed, GE Medical Systems, Milwaukee, WI, USA) for brachytherapy planning. All patients followed the bladder filling protocol with $50-100 \mathrm{ml}$ saline before the MRI and CT scans. The images were exported to brachytherapy planning system (BrachyVision version 13.6, Varian Medical Systems, Palo Alto, CA, USA). All target volumes and OAR delineations were performed on both image modalities by a same radiation oncologist.

\section{Treatment planning}

The treatment plans were generated for three different imaging schemes (Figure 1), in MRI-only approach in $\mathrm{MRI}_{1 \mathrm{st}} / \mathrm{CT}$ approach, and in CT-only approach. The MRI-only scheme was used as a standard approach. In MRI-only, the high-risk clinical target volume (HRCTV) and all OAR (bladder, rectum, sigmoid colon, and small bowel) on each fraction were contoured on the MRI, as recommended by GEC-ESTRO. In $\mathrm{MRI}_{1 \mathrm{st}} / \mathrm{CT}$, images from the first fraction (MRI) were registered to the subsequent $\mathrm{CT}$ images (second to fourth fractions) using image regis- tration software in the treatment planning system. Then, the HRCTV on the first fraction's MRI was transferred to the corresponding subsequent $\mathrm{CT}$ with adjustment to the CT images. In CT-only, the HRCTV and all OAR were drawn on the CT images. To avoid bias in the contouring for CT-only, the HRCTV was delineated on the CT images by a single radiation oncologist before being contoured on the MRI.

After the HRCTV and all OAR delineations were completed, treatment plans were generated. On the MRI- and CT-based plans, the applicator reconstruction and dose optimization were done via a manual technique, using the TG-43 dose calculation algorithm to compute the desired dose distribution. The dwell times and location of the radioactive source were optimized for prescribed doses $7 \mathrm{~Gy}$ to $\mathrm{D}_{90}$ of the HRCTV, with the total target dose from external beam RT and BT $\geq 84 \mathrm{~Gy}$, in 2 Gy equivalent (EQD2) and similar acceptable doses to the OAR in the same fraction for each planning approach to be $\leq 90$ EQD2 for $D_{2 c c}$ bladder, $\leq 70$ for $D_{2 c c}$ rectum and sigmoid as suggested by GEC-ESTRO Working Group II [19].

\section{Data collection and statistical analyses}

The volume, height, width at point $\mathrm{A}$, width at the maximum level as well as the dosimetric parameters $\left(\mathrm{D}_{100}, \mathrm{D}_{98}, \mathrm{D}_{95}\right.$, and $\mathrm{D}_{90}$ of the HRCTV; and $\mathrm{D}_{0.1 \mathrm{cc}}, \mathrm{D}_{1 \mathrm{cc}}$ and $\mathrm{D}_{2 c c}$ of all OAR) were collected from the dose-volume histogram and analyzed. The data were reported as averages (mean and SD). Repeated measure ANOVA was used to compare the differences between each fraction of parameters. Differences with a $p$ value $<0.05$ were considered to be statistically significant. All calculations were performed with SPSS Statistics for Windows, version 16.0 (SPSS Inc, Chicago, Ill, USA).

\section{Results}

\section{HRCTV volume and dimension}

The HRCTV volume from MRI of the 10 patients in this study was in the range of 11.9-30.2 cc (mean $=18.8$, $\mathrm{SD}=6.95$ ). From Table 1, statistically significant ratios of the HRCTV CT to the MRI were 1.48 for the volume, 1.20 for the width at point $\mathrm{A}$, and 1.21 for the height. Using $\mathrm{MRI}_{1 \mathrm{st}} / \mathrm{CT}$, the ratios of the overestimated volume, width, and height (fraction 2-4) were reduced to 1.11, 1.12 , and 1.07, respectively. As to fraction-by-fraction comparisons, no differences were detected between MRI

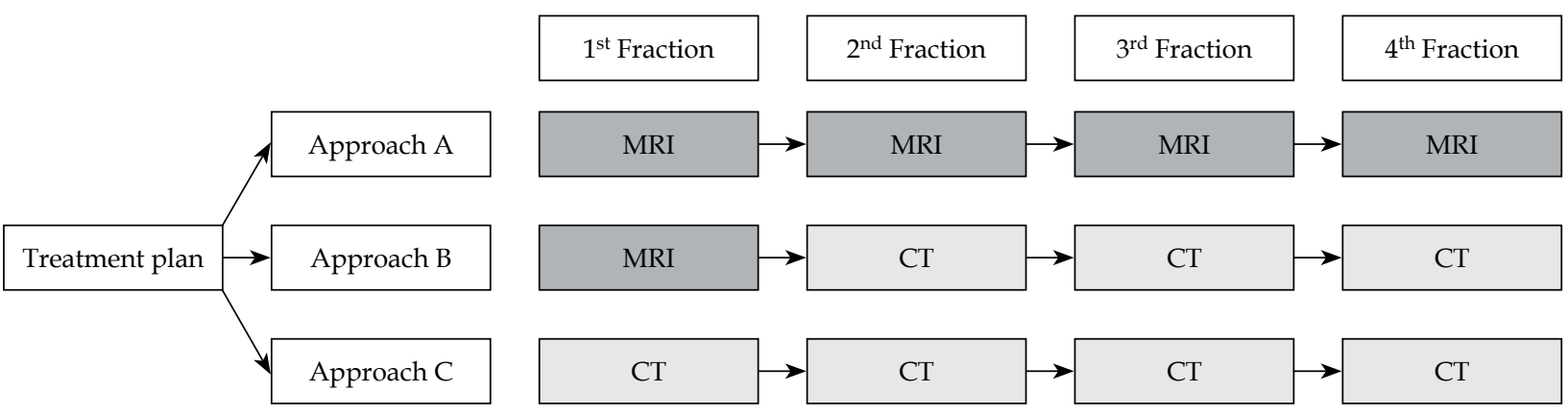

Fig. 1. Schematic workflow of the three imaging approaches 
and $\mathrm{MRI}_{1 \mathrm{st}} / \mathrm{CT}$ in terms of the total volume, the width at the maximum level, the width at point $\mathrm{A}$, and the height (Table 2). However, the HRCTV volume, including the width at point $\mathrm{A}$ and the height obtained from $\mathrm{CT}$, was significantly larger than that from MRI. Also, similar results of volume and height were shown for comparisons of the $\mathrm{MRI}_{1 \mathrm{st}} / \mathrm{CT}$ and $\mathrm{CT}$ approaches.

\section{Dosimetric parameters}

The dosimetric parameters for the HRCTV and all OAR are presented in Tables 3 and 4 . There were no differences in the doses to the HRCTV for approaches A and B. In contrast, the doses to the HRCTV for approach $C$ were significantly lower than those for MRI-only for all parameters $\left(D_{95}-D_{100}\right)$. Similar results were shown for the comparisons of approaches B and C. As to the OAR doses, there were no differences between the three approaches.

\section{Discussion}

It has been confirmed that 3D IGBT using MRI- or CTbased planning offers a better assessment of tumor volume than 2D-based planning. Their accuracy of the target volume and all OAR delineations effectively deliver more tumor-specific high doses, with less adverse effects on the surrounding normal tissues $[3,4,20,21,22,23,24,25,26]$. However, with CT, it is very difficult to identify the cervix and parametrium, and cause the tumor volume delineation to be overestimated, which results in a reduced dose coverage for the tumor volume and increased doses to OAR $[7,8,9,10,26,27]$. On the other hand, MRI-based planning provides better accuracy for the HRCTV and all OAR delineations. Superior treatment outcomes with impact in local control and survival from MRI-based planning have been presented by various groups [15,23,24,28,29]. However, the relative lack of availability of this superior modality is a disadvantage. Therefore, the combination of $\mathrm{MRI}_{1 \mathrm{st}} /$ CT technique was introduced by Beriwal et al. [17]. The study showed that this approach is feasible and has excellent local control. A similar dose coverage to the HRCTV (especially in case of a small tumor volume) to that provided by fully MRI-based planning has also been reported by Nesvacil et al. [18].
Regarding the difference in the target volume using CT and MRI for brachytherapy for cervical cancer, Swanick et al. [30] studied the HRCTV volume using two image modalities, and showed that the mean HRCTV volume obtained using CT $\left(44.1 \mathrm{~cm}^{3}\right)$ was larger than that obtained using MRI $\left(35.1 \mathrm{~cm}^{3}\right)$, with statistical significance $(p<0.0001)$. Due to increased discrepancy in the HRCTV volume obtained with MRI, they concluded that MRIbased brachytherapy planning should be considered for the use in patients with a higher body mass index and a tumor size of $5 \mathrm{~cm}$ or greater, with parametrial invasion.

In the present study, the HRCTV volume obtained from fully CT-based planning was overestimated compared to the volumes acquired using full MRI-based and $\mathrm{MRI}_{1 \mathrm{st}} / \mathrm{CT}$ approaches. As a consequence, the HRCTV doses obtained from CT-based planning were lower than those provided by the other two approaches in almost every fraction, and the range of dose differences were larger for the higher doses to the target (e.g., for approaches $\mathrm{A}$ and C: $\mathrm{D}_{90 \mathrm{cc}} 0.69-0.83$ Gy and $D_{100 c c} 0.75-1.20 \mathrm{~Gy}$, respectively). These outcomes are very similar to those in Nesvacil study [18], and it can be concluded that the overestimations on the CT images can be overcome by using MRI in the $1^{\text {st }}$ fraction to guide contouring on the subsequent fractions of the CT images. This outcome was also confirmed by the results of better the HRCTV volumes and dimensions, and improved doses for approach B than approach $C$, even though all were obtained from CT images. A study by Choong et al. [31] analyzed 76 cervical cancer patients who had been divided into 49 cases with CT- and MRI-guided brachytherapy (the same as approach B in the present study) and 27 cases with MRI-guided only brachytherapy (the same as MRI-only in this study). The results showed no statistically significant differences in the clinical outcomes or dosimetric analyses of the two approaches. Wang et al. [32] reviewed 13 clinical studies comprising a total of 465 patients to evaluate the accuracy of two image modalities for the brachytherapy of cervical cancer. Ten studies compared CT to MRI, while the other three compared $\mathrm{MRI}_{1 \mathrm{st}} / \mathrm{CT}$ to MRI-based imaging; however, none of these studies compared all three imaging approaches on one patient (unlike the current study). Wang et al. found that, compared to MRI, the HRCTV width had been overestimated, while the height might have been underestimated when using CT. The dosimetric analyses

Table 1. Overall results of the HRCTV volume and dimensions for the ten patients

\begin{tabular}{|c|c|c|c|c|c|c|c|c|}
\hline \multirow[t]{2}{*}{ HRCTV } & \multirow[t]{2}{*}{$\mathrm{Fr}$} & \multirow{2}{*}{$\frac{\text { MRI }(\mathrm{A})}{\text { Mean } \pm \text { SD }}$} & \multirow{2}{*}{$\frac{\mathrm{MRI}_{1 \mathrm{st}} / \mathrm{CT}(\mathrm{B})}{\text { Mean } \pm \mathrm{SD}}$} & \multirow{2}{*}{$\frac{\mathrm{CT}(\mathrm{C})}{\text { Mean } \pm \mathrm{SD}}$} & \multicolumn{4}{|c|}{ Ratio } \\
\hline & & & & & A vs. B & $p$-value & A vs. C & $p$-value \\
\hline \multirow[t]{2}{*}{ Volume } & $1-4$ & $18.8 \pm 6.7$ & - & $27.7 \pm 10.1$ & - & - & 1.48 & $0.00^{*}$ \\
\hline & $2-4$ & $18.6 \pm 6.8$ & $20.8 \pm 6.8$ & $26.7 \pm 9.3$ & 1.11 & $0.02^{*}$ & 1.47 & $0.00^{*}$ \\
\hline \multirow{2}{*}{$\begin{array}{l}\text { Width } \\
\text { (maximum) }\end{array}$} & $1-4$ & $4.2 \pm 0.4$ & - & $4.4 \pm 0.6$ & - & - & 1.06 & 0.04 \\
\hline & $2-4$ & $4.1 \pm 0.4$ & $4.1 \pm 0.5$ & $4.3 \pm 0.4$ & 0.98 & 0.78 & 1.05 & 0.09 \\
\hline \multirow{2}{*}{$\begin{array}{l}\text { Width } \\
\text { (point A) }\end{array}$} & 1 & $2.7 \pm 0.7$ & - & $3.2 \pm 0.7$ & - & - & 1.20 & $0.01^{*}$ \\
\hline & $2-4$ & $2.5 \pm 0.7$ & $2.8 \pm 0.7$ & $3.1 \pm 0.7$ & 1.12 & 0.08 & 1.18 & $0.05^{*}$ \\
\hline \multirow[t]{2}{*}{ Height } & 1 & $2.7 \pm 0.7$ & - & $3.3 \pm 0.4$ & - & - & 1.21 & $0.01^{*}$ \\
\hline & $2-4$ & $2.8 \pm 0.7$ & $2.9 \pm 0.6$ & $3.3 \pm 0.4$ & 1.07 & 0.48 & 1.22 & $0.02^{*}$ \\
\hline
\end{tabular}

HRCTV - high-risk clinical target volume; MRI - magnetic resonance imaging; CT - computed tomography; Fr - fraction; SD - standard deviation; *statistically significant $(p<0.05)$ 







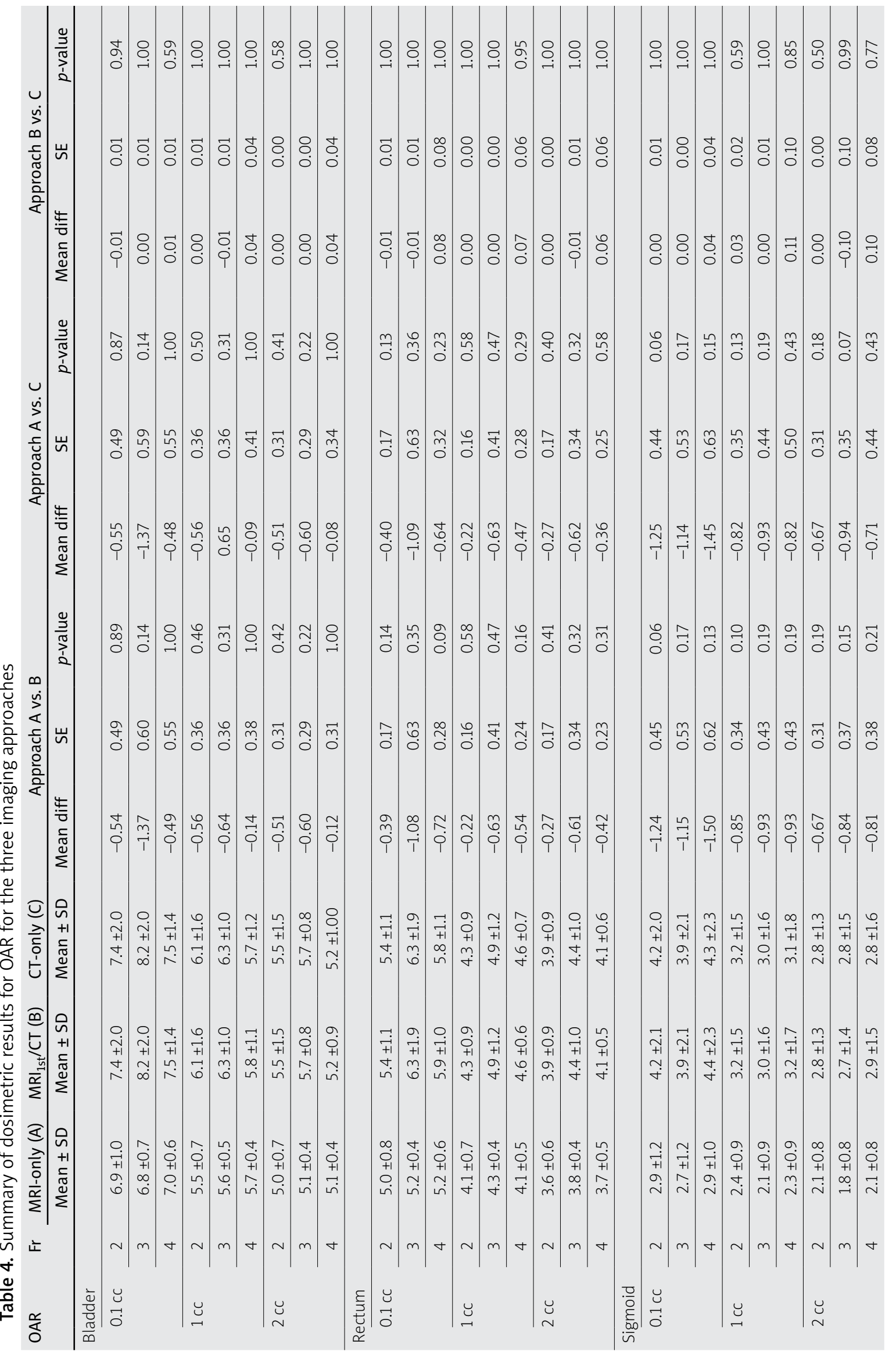




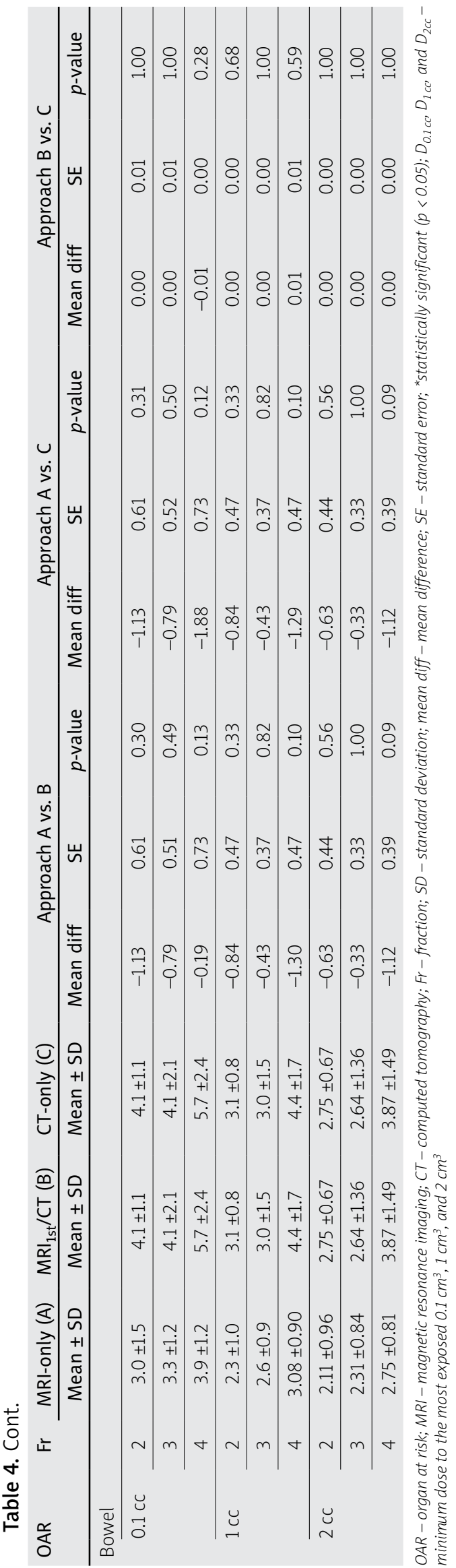

for the HRCTV were also lower for the CT-only approach compared with at least one fraction of the MRI-based brachytherapy. These outcomes were similar to the results of the current study. However, the height of the HRCTV obtained from our CT approach was contoured at two-thirds of the uterine height, as suggested by Hegazy et al. [33] and was found to be significantly higher compared to the results obtained with the MRI-only and the $\mathrm{MRI}_{1 \mathrm{st}} / \mathrm{CT}$ approaches. As to fraction-by-fraction comparisons, there was a significant overestimation of the volume, and the dimension was obtained from the CT modality.

With regards to doses delivered to OAR, most dosimetric parameters in the dose-volume histogram, including $\mathrm{D}_{0.1 \mathrm{cc}}, \mathrm{D}_{1 \mathrm{cc},}$ and $\mathrm{D}_{2 \mathrm{cc}}$ showed no differences for the three imaging approaches used in the present study. In comparison, the Wang study [33] demonstrated that there were no statistically significant differences between the two modalities for the dosimetric analysis of OAR conducted in 4 studies. Furthermore, the MRI-guided brachytherapy provided an improved dose to the bladder in 4 studies, to the rectum in 4 other studies, and to the sigmoid in the remaining study. However, the clinical toxicity incidence showed no statistically significant difference between the two modalities.

The results of the present study indicate that the use of MRI-guided brachytherapy for target localization in $\mathrm{MRI}_{1 \mathrm{st}} / \mathrm{CT}$ approach in cervical cancer brachytherapy provides an improved accuracy and precision of the tumor volume as well as a better dose coverage to the HRCTV, when it is compared with CT exclusive-based planning. At least one MRI is required to assess the tumor extension due to the overestimation of the HRCTV volume obtained using CT images (which was similar to the findings of other studies). However, there are some limitations of this study: it included small number of patients, and the interobserver variation was not assessed.

\section{Conclusions}

$\mathrm{MRI}_{1 \mathrm{st}} / \mathrm{CT}$ approach is a safe alternative for brachytherapy in cervical cancer patients, especially in those centers with limited MRI availability. Moreover, it is more cost-effective than the MRI-based approach.

\section{Acknowledgements}

We are very grateful to David Park for proofreading this manuscript.

\section{Disclosure}

Authors report no conflict of interest.

\section{References}

1. Mayadev J, Viswanathan A, Liu Y et al. American Brachytherapy Task Group Report: A pooled analysis of clinical outcomes for high-dose-rate brachytherapy for cervical cancer. Brachytherapy 2017; 16: 22-43.

2. Pötter R, Georg P, Dimopoulos JC et al. Clinical outcome of protocol-based image (MRI) guided adaptive brachytherapy combined with 3D conformal radiotherapy with or without chemotherapy in patients with locally advanced cervical cancer. Radiother Oncol 2011; 100: 116-123. 
3. Datta NR, Srivastava A, Maria Das KJ et al. Comparative assessment of doses to tumor, rectum, and bladder as evaluated by orthogonal radiographs vs. computer enhanced computed tomography-based intracavitary brachytherapy in cervical cancer. Brachytherapy 2006; 5: 223-229.

4. Shin KH, Kim TH, Cho JK et al. CT-guided intracavitary radiotherapy for cervical cancer: Comparison of conventional point A plan with clinical target volume-based three-dimensional plan using dose volume parameters. Int J Radiat Oncol Biol Phys 2006; 64: 197-204.

5. Pelloski CE, Palmer M, Chronowski GM et al. Comparison between CT-based volumetric calculations and ICRU reference-point estimates of radiation doses delivered to bladder and rectum during intracavitary radiotherapy for cervical cancer. Int J Radiat Oncol Biol Phys 2005; 62: 131-137.

6. Tyagi K, Mukundan H, Mukherjee D et al. Non isocentric film-based intracavitary brachytherapy planning in cervical cancer: a retrospective dosimetric analysis with CT planning. J Contemp Brachytherapy 2012; 3: 129-134.

7. Viswanathan A, Dimopoulos J, Kirisits C et al. Computed tomography versus magnetic resonance imaging-based contouring in cervical cancer brachytherapy: results of a prospective trial and preliminary guidelines for standardized contours. Int J Radiat Oncol Biol Phys 2007; 68: 491-498.

8. Mitchell DG, Snyder B, Coakley F et al. Early invasive cervical cancer: tumor delineation by magnetic resonance imaging, computed tomography, and clinical examination, verified by pathologic results, in the ACRIN 6651/GOG 183 Intergroup Study. J Clin Oncol 2006; 24: 5687-5694.

9. Ozsarlak O, Tjalma W, Schepens E et al. The correlation of preoperative $\mathrm{CT}, \mathrm{MR}$ imaging, and clinical staging (FIGO) with histopathology findings in primary cervical carcinoma. Eur Radiol 2003; 13: 2338-2345.

10. Bipat S, Glas AS, van der Velden J et al. Computed tomography and magnetic resonance imaging in staging of uterine cervical carcinoma: a systematic review. Gynecol Oncol 2003; 91: 59-66.

11. Hricak H, Gatsonis C, Coakley FV et al. Early invasive cervical cancer: CT and MR imaging in preoperative evaluation ACRIN/GOG comparative study of diagnostic performance and interobserver variability. Radiology 2007; 245: 491-498.

12. Haie-Meder C, Pötter R, Van Limbergen E et al. Recommendations from Gynaecological (GYN) GEC-ESTRO Working Group (I): concepts and terms in 3D image-based 3D treatment planning in cervix cancer brachytherapy with emphasis on MRI assessment of GTV and CTV. Radiother Oncol 2005; 74: 235-245.

13. Dimopoulos J, Petrow P, Tanderup K et al. Recommendations from Gynaecological (GYN) GEC-ESTRO Working Group (IV): Basic principles and parameters for MR imaging within the frame of image based adaptive cervix cancer brachytherapy. Radiother Oncol 2012; 103: 113-122.

14. EMBRACE: www.embracestudy.dk

15. Sturdza A, Pötter R, Fokdal LU et al. Image guided brachytherapy in locally advanced cervical cancer: Improved pelvic control and survival in RetroEMBRACE, a multicenter cohort study. Radiother Oncol 2016; 120: 428-433.

16. Harkenrider M, Shea S, Wood A et al. How one institution overcame the challenges to start an MRI-based brachytherapy program for cervical cancer. J Contemp Brachytherapy 2017; 9: 177-186.

17. Beriwal S, Kannan N, Kim H et al. Three-dimensional high dose rate intracavitary image-guided brachytherapy for the treatment of cervical cancer using a hybrid magnetic resonance imaging/computed tomography approach: feasibility and early results. Clin Oncol 2011; 23: 685-690.

18. Nesvacil N, Pötter R, Sturdza A et al. Adaptive image guided brachytherapy for cervical cancer: a combined MRI-/CT-plan- ning technique with MRI only at first fraction. Radiother Oncol 2013; 107: 75-81.

19. Pötter R, Haie-Meder C, Van Limbergen E et al. Recommendations from gynaecological (GYN) GEC-ESTRO Working Group (II): concepts and terms in 3D image-based treatment planning in cervix cancer brachytherapy-3D dose volume parameters and aspects of 3D image-based anatomy, radiation physics, radiobiology. Radiother Oncol 2006; 78: 67-77.

20. Tan LT, Coles CE, Hart C, Tait E. Clinical impact of computed tomography-based image-guided brachytherapy for cervix cancer using the tandem-ring applicator-the Addenbrooke's experience. Clin Oncol 2009; 21: 175-182.

21. Lindegaard JC, Tanderup K, Nielsen SK et al. MRI-guided 3D optimization significantly improves DVH parameters of pulsed-dose-rate brachytherapy in locally advanced cervical cancer. Int J Radiat Oncol Biol Phys 2008; 71: 756-764.

22. Chargari C, Magné N, Dumas I et al. Physics contributions and clinical outcome with 3D-MRI-based pulsed-dose-rate intracavitary brachytherapy in cervical cancer patients. Int J Radiat Oncol Biol Phys 2009; 74: 133-139.

23. Pötter R, Dimopoulos J, Georg P et al. Clinical impact of MRI assisted dose volume adaptation and dose escalation in brachytherapy of locally advanced cervix cancer. Radiother Oncol 2007; 83: 148-155.

24. Lindegaard JC, Fokdal LU, Nielsen SK et al. MRI-guided adaptive radiotherapy in locally advanced cervical cancer from a Nordic perspective. Acta Oncol 2013; 52: 1510-1519.

25. Šegedin B, Gugić J, Petrič P. Uterine perforation - 5-year experience on 3D image guided gynaecological brachytherapy at Institute of Oncology Ljubljana. Radiol Oncol 2013; 47: 154-160.

26. Derks K, Steenhuijsen J, van den Berg $\mathrm{H}$ et al. Impact of brachytherapy technique (2D versus $3 \mathrm{D})$ on outcome following radiotherapy of cervical cancer. J Contemp Brachytherapy 2018; 10: 17-25.

27. Yip W, Wong J, Lee $\mathrm{V}$ et al. Throwing the dart blind-folded: comparison of computed tomography versus magnetic resonance imaging-guided brachytherapy for cervical cancer with regard to dose received by the 'actual' targets and organs at risk. J Contemp Brachytherapy 2017; 9: 446-452.

28. Tanderup K, Viswanathan AN, Kirisits C, Frank SJ. Magnetic resonance image guided brachytherapy. Semin Radiat Oncol 2014; 24: 181-191.

29. Pötter R, Georg P, Dimopoulos J, Grimm M et al. Clinical outcome of protocol-based image (MRI) guided adaptive brachytherapy combined with 3D conformal radiotherapy with or without chemotherapy in patients with locally advanced cervical cancer. Radiother Oncol 2011; 100: 116-123.

30. Swanick CW, Castle KO, Vedam S et al. Comparison of computed tomography and magnetic resonance imaging-based clinical target volume contours at brachytherapy for cervical cancer. Int J Radiat Oncol Biol Phys 2016; 96: 793-800.

31. Choong ES, Bownes P, Musunuru HB et al. Hybrid (CT/MRI based) vs. MRI only based image-guided brachytherapy in cervical cancer: Dosimetry comparisons and clinical outcome. Brachytherapy 2016; 15: 40-48.

32. Wang F, Tang Q, Lv G et al. Comparison of computed tomography and magnetic resonance imaging in cervical cancer brachytherapy: a systemic review. Brachytherapy 2017; 16: 353-365.

33. Hegazy N, Potter R, Kirisits C et al. High-risk clinical target volume delineation in CT-guided cervical cancer brachytherapy: Impact of information from FIGO stage with or without systematic inclusion of 3D documentation of clinical gynecological examination. Acta Oncol 2013; 52: 1345-1352. 